\title{
Protein-protein interaction between caveolin-1 and SHP-2 is dependent on the N-SH2 domain of SHP-2
}

\author{
Hyunju Park ${ }^{1}$, Keun Jae Ahn ${ }^{2}$, Jihee Lee Kang ${ }^{1} \mathcal{E}$ Youn-Hee Choi ${ }^{1, *}$ \\ ${ }^{1}$ Department of Physiology, Tissue Injury Defense Research Center, Ewha Womans University School of Medicine, Seoul 158-710, \\ ${ }^{2}$ Department of Science Education, Jeju National University, Jeju 690-756, Korea
}

Src homology 2-containing protein tyrosine phosphatase 2 (SHP-2) is known to protect neurons from neurodegeneration during ischemia/reperfusion injury. We recently reported that ROS-mediated oxidative stress promotes phosphorylation of endogenous SHP-2 in astrocytes and complex formation between caveolin-1 and SHP-2 in response to oxidative stress. To examine the region of SHP-2 participating in complex formation with caveolin-1, we generated three deletion mutant constructs and six point mutation constructs of SHP-2. Compared with wild-type SHP-2, binding of the N-SH2 domain deletion mutant of SHP-2 to p-caveolin-1 was reduced greatly, using flow cytometric competitive binding assays and surface plasmon resonance (SPR). Moreover, deletion of the $\mathrm{N}-\mathrm{SH} 2$ domain of SHP-2 affected $\mathrm{H}_{2} \mathrm{O}_{2}$-mediated ERK phosphorylation and Src phosphorylation at Tyr 419 in primary astrocytes, suggesting that N-SH2 domain of SHP-2 is responsible for the binding of caveolin-1 and contributes to the regulation of Src phosphorylation and activation following ROS-induced oxidative stress in brain astrocytes. [BMB Reports 2015; 48(3): 184-189]

\section{INTRODUCTION}

Oxidative stress due to accumulation of reactive oxygen species (ROS) is involved in cell death associated with neurological disorders, such as stroke, traumatic brain injury, and Alzheimer's disease (1-3). ROS and reactive nitrogen species (RNS) change cellular responses through diverse molecular mechanisms. At high levels, ROS and RNS damage organelles, through iron ion- or copper ion-mediated oxidation of lipids, proteins, and nucleic acids (4). Astrocytes are the most abun-

*Corresponding author. Tel: +82-2-2650-5838; Fax: +82-2-26505717; E-mail: yc@ewha.ac.kr

http://dx.doi.org/10.5483/BMBRep.2015.48.3.249

Received 21 November 2014, Revised 6 December 2014, Accepted 4 February 2015

Keywords: Astrocytes, Caveolin-1, Reactive oxygen species, SHP-2, Src dant cell type in the brain and play multiple roles in diverse functions, including protection against metal toxicity and oxidative stress (5-8). However, how brain cells, including astrocytes, survive in an environment with continuous ROS exposure and whether brain cells possess specific defense mechanisms against ROS are poorly understood.

Several recent studies have demonstrated that protein phosphatases play a neuroprotective role against oxidative stress in response to ischemic brain injury (9). Recently, we reported that Src homology 2 containing protein tyrosine phosphatase 2 (SHP-2, also known as PTPN11), one of cytoplasmic protein-tyrosine phosphatases, functions as a component of the raft-mediated signaling pathway that acts through complex formation with caveolin-1 and regulation of a signaling cascade upon ROS-induced oxidative stress $(10,11)$. SHP-2 contains two tandem Src homology $2(\mathrm{SH} 2)$ domains, a tyrosine phosphatase domain, and a carboxyl-terminal tail that contains two tyrosyl phosphorylation sites (12). Although SHP-2 is a cytoplasmic phosphatase, existing mainly in the cytosol, the enzyme is also known to translocate to its target molecules on lipid rafts, and then acts as either a positive or negative regulator in numerous signaling pathways (13-15).

Caveolin-1, a 21-24-kDa membrane protein, is a multifunctional scaffold protein that harbors binding sites for multiple binding partners and is associated with cell surface caveolae and the regulation of lipid raft domains $(16,17)$. Src, Abl, and Fyn phosphorylate caveolin- 1 on its Tyr 14 residue in response to various stimuli, including insulin, oxidative stress, and angiotensin II osmotic shock (18). Tyrosine phosphorylation at the 14 residue of caveolin-1 plays an important role in providing a docking site to anchor various proteins, regulating several signaling molecules and modulating downstream signaling pathways, negatively or positively $(19,20)$. Our recent studies suggested that $\mathrm{H}_{2} \mathrm{O}_{2}$-mediated oxidative stress induces phosphorylation of caveolin-1 and complex formation of caveolin-1 and SHP-2, and that caveolin-1 is involved in the ROS-induced activation of SHP-2 (11). However, which domain of SHP-2 is responsible for the binding of caveolin- 1 and for modulating downstream signal transduction in response to ROS-induced oxidative stress remain unclear. Thus, in this study, we examined the binding affinity of wildtype and mutant SHP-2 proteins to phosphorylated caveolin-1 and changes

ISSN: 1976-670X (electronic edition)

Copyright (C) 2015 by the The Korean Society for Biochemistry and Molecular Biology

(c) This is an open-access article distributed under the terms of the Creative Commons Attribution Non-Commercial License (http://creativecommons.org/licenses/by-nc/3.0) which permits unrestricted non-commercial use, distribution, and reproduction in any medium, provided the original work is properly cited. 
in Src kinase and ERK activity following transfection of wild-type and mutant SHP-2 under $\mathrm{H}_{2} \mathrm{O}_{2}$-mediated oxidative stress.

\section{RESULTS}

\section{Preparation of wild-type and mutant SHP-2 proteins}

SHP-2 contains two N-terminal $\mathrm{SH} 2$ domains $(\mathrm{N}-\mathrm{SH} 2$ and $\mathrm{C}-\mathrm{SH} 2$ ), a tyrosine phosphatase domain (PTP), and a C-terminal tail that contains two tyrosyl phosphorylation sites (12). To determine the region of SHP-2 responsible for the binding of caveolin-1, we generated three deletion mutants of the $\mathrm{SH} 2$ domains and six point mutants, as described in the Materials and Methods (Fig. 1A). Recombinant GST-wild-type SHP-2 (rGST-WT-SHP-2) and mutant SHP-2 proteins were purified using glutathione affinity chromatography columns and were assessed using sodium dodecyl sulfate-polyacrylamide gel electrophoresis (SDS-PAGE). Purified rGST-WT-SHP-2 appeared as a 95-kDa band on the SDS-PAGE gel, whereas purified rGST-mutant-SHP-2 appeared as 70-95-kDa bands (Fig. 1B). The SDSPAGE results demonstrated that all ten proteins were purified qualitatively with high purity. The purity and homogeneity of the purified recombinant proteins were analyzed by Western blot analysis. The N-SH2 domain deletion mutant SHP-2 (SHP-2 $\Delta 6$-102) and $\mathrm{N}-\mathrm{SH} 2$ and $\mathrm{C}-\mathrm{SH} 2$ domain deletion mutant SHP-2 (SHP-2 $\Delta$ double) were not detected by anti-SHP-2, which recognizes amino acid 1-177. On the other hand, an
anti-GST antibody recognized all the wild-type and mutant SHP-2 proteins, and showed that the expression of the mutant SHP-2 proteins was comparable (Fig. 1C).

\section{Characterization of binding affinity of wild-type and mutant SHP-2 proteins to p-caveolin-1}

We previously reported that phosphorylation of the Tyr 14 residue of caveolin-1 is required for complex formation between caveolin-1 and SHP-2 in rat primary astrocytes and human astroglioma cells. To determine which domain of the SHP-2 bound to the p-Y14 site of caveolin-1, a bead-based flow cytometric binding assay was performed. Conjugation of a p-Y14 caveolin-1 peptide (aa 1-50) to carboxyl beads was conducted using 1-ethyl-3-(3-dimethylaminopropyl) carbodiimide (EDC) as described in the Materials and Methods. Caveolin-1 peptide-conjugated beads were incubated with $10 \mu \mathrm{g}$ of recombinant wild-type or mutant SHP-2 proteins. After washing with ice-cold PBS, beads were incubated with anti-SHP-2 and anti-GST antibody and then with Alexa 488-conjugated goat anti-mouse Ig antibody and FITC-conjugated goat anti-rabbit IG antibody. The samples were analyzed by flow cytometry. Histograms include levels of mean fluorescence intensity (MFI) ratio of SHP-2 binding to p-caveolin-1. Compared with the wild-type SHP-2, the $\mathrm{N}-\mathrm{SH} 2$ domain deletion mutant SHP-2 (SHP-2 $\Delta 6$-102) was not bound to p-caveolin-1 (Fig. 2A, B). Binding of the $\mathrm{C}-\mathrm{SH} 2$ domain deletion mutant and point mutations in the PTP domains to p-caveolin-1 was reduced slightly
A

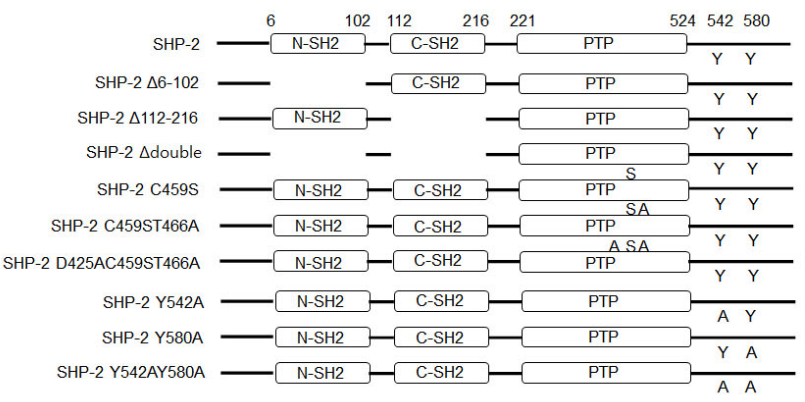

B

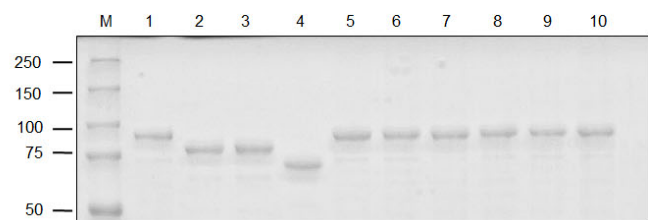

c

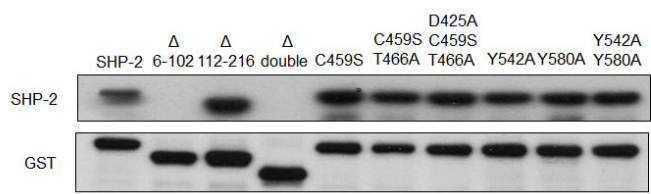

Fig. 1. Preparation of rGST-WT-SHP-2 and rGST-mutants-SHP-2. (A) Diagram of the wild-type and mutant SHP-2 constructs. SHP-2 consists of the two $\mathrm{SH} 2$ domains ( $\mathrm{N}-\mathrm{SH} 2$ and $\mathrm{C}-\mathrm{SH} 2$ ), a catalytic protein-tyrosine phosphatase (PTP) domain, and a C-terminal tail. (A, alanine; $\mathrm{C}$, cysteine; $\mathrm{S}$, serine; $\mathrm{T}$, threonine; D, aspartic acid; $\mathrm{Y}$, tyrosine) (B) The purified proteins were analyzed on a $10 \%$ SDS-polyacrylamide gel, and the wild-type and mutant SHP-2 protein bands were stained with Coomassie Brilliant Blue R250. (C) Protein levels of wild-type and mutant SHP-2. The blots were subjected to immunoblot analysis with anti-SHP-2 and anti-GST antibodies. 
A
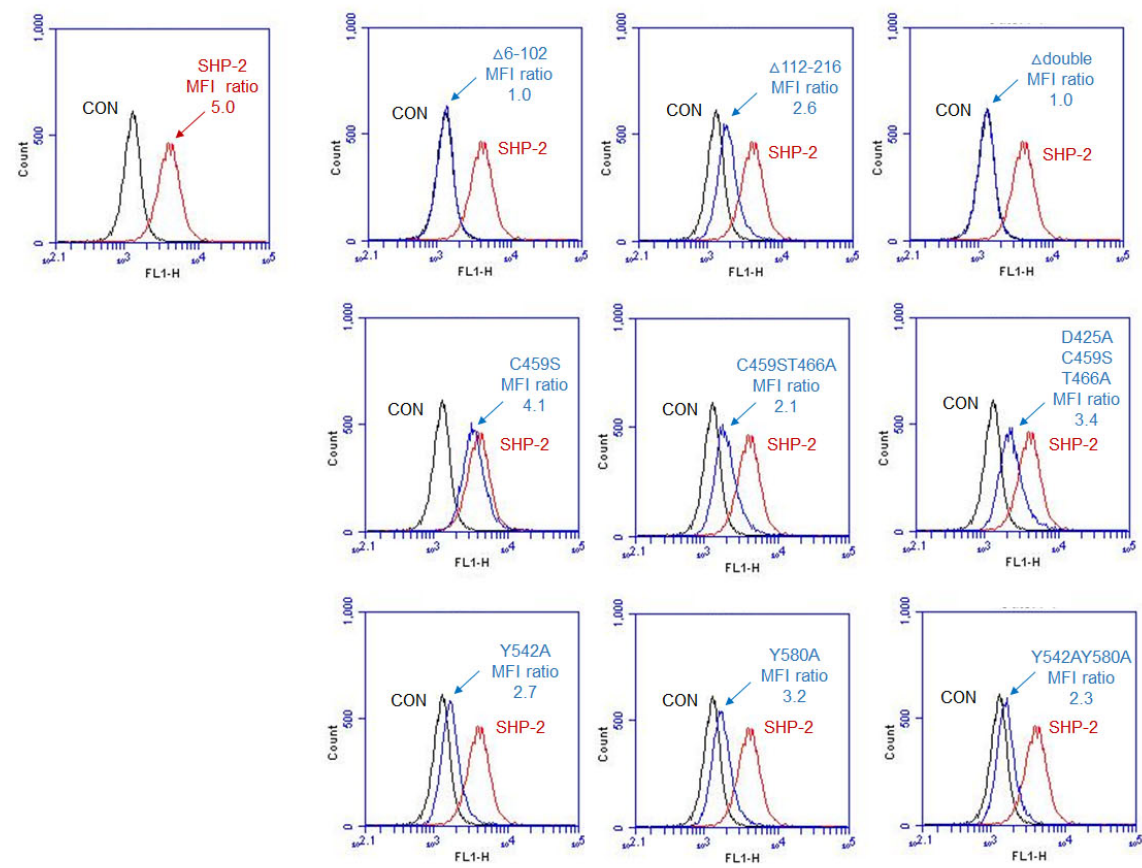

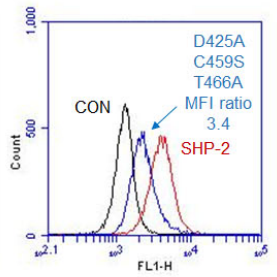

B
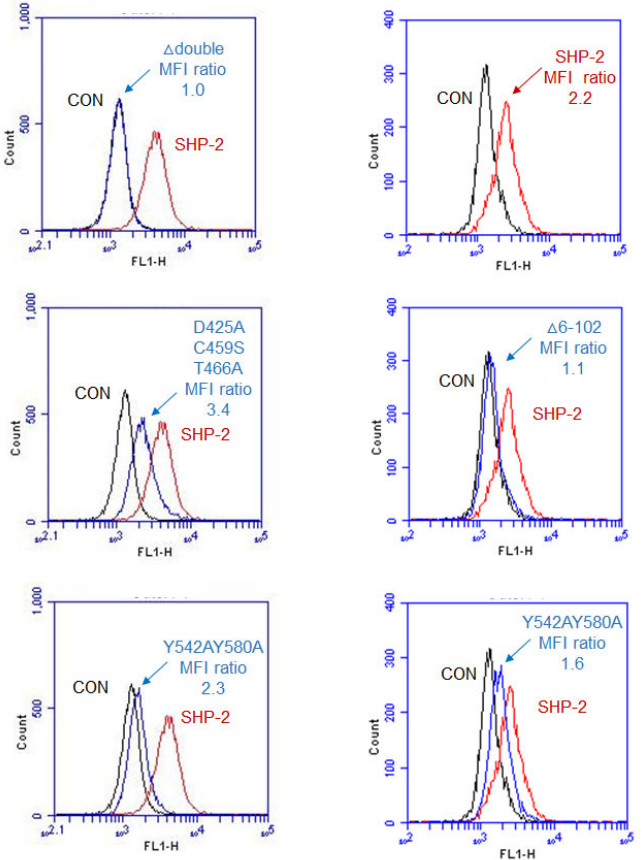

Fig. 2. Flow cytometric analyses of binding affinity of wild-type and mutant SHP-2 to phosphorylated-Tyr peptide of caveolin-1. A synthetic peptide of caveolin-1 (aa 1-50, phosphorylation at the tyrosine 14 residue) was used in this study. Flow cytometric binding assay was performed by incubating bead-conjugated caveolin-1 peptide with $10 \mu \mathrm{g}$ of wild-type or mutant SHP-2 proteins. After washing with ice-cold PBS, beads were incubated with $1 \mu \mathrm{g}$ of anti-SHP-2 (A) and anti-GST (B) antibodies and then incubated with Alexa 488-conjugated IgG and FITC-conjugated IgG antibodies. After stringent washes, SHP-2 bound to caveolin-1 peptide-conjugated beads was measured with an Accuri C6 flow cytometer. Numbers in the graph refer to the mean fluorescent intensity (MFI) ratio. The experiments were repeated three times and similar results were obtained.

or comparable to wild-type SHP-2. The binding affinity of SHP-2 with the point mutations in the C-terminal tail containing the tyrosyl phosphorylation sites to p-caveolin-1 was also reduced versus that of wild-type SHP-2. These results indicated that the $\mathrm{N}-\mathrm{SH} 2$ domain of SHP-2 plays a key role in complex formation between caveolin-1 and SHP-2 in response to $\mathrm{H}_{2} \mathrm{O}_{2}$-mediated oxidative stress.

\section{SPR analyses of wild-type and mutant SHP-2 binding to p-caveolin-1}

To confirm the binding pattern of wild-type and mutant SHP-2 to phosphorylated caveolin-1, SPR experiments were performed using a ProteOn XPR36 system (Bio-Rad) as described in the Materials and Methods. Caveolin-1 peptide phosphorylated at the tyrosine 14 residue immobilized on a GLH sensor chip was used to determine the binding kinetics of wild-type and mutant SHP-2 proteins. As shown in Fig. 3, wild-type SHP-2 was found to bind to p-Y14 caveolin-1 with high affinity. On the other hand, the RU value of deletion of $\mathrm{N}-\mathrm{SH} 2$ domain of mutants SHP-2 (SHP-2 $\Delta 6-102)$ was reduced sig- nificantly compared with the wild-type SHP-2, indicating that the binding affinity was decreased significantly by mutations, consistent with the bead-based flow cytometric binding assay. Also, data on the protein dissociation rate were obtained by measuring the amount of protein bound while rinsing the chip with buffer. The remaining proteins were measured at two time points: dissociation 1 (150 s) and dissociation 2 (250 s; Fig. 3). The binding kinetics of the C459S mutant SHP-2 to p-caveolin-1 were similar to those of the wild-type SHP-2; however, the kinetic rates or resonance units (RU) of the Y542AY580A mutant SHP-2 were also reduced versus the wild-type SHP-2.

\section{Effects of wild-type and mutant SHP-2 on $\mathrm{H}_{2} \mathrm{O}_{2}$-induced Src phosphorylation}

To determine whether wild-type and mutant SHP-2 affected Src activation and phosphorylation under oxidative stress in primary astrocytes, a protein transfection assay was performed using the Pro-Ject Protein Transfection Reagent Kit (Thermo Scientific). Cells were transfected with wild-type or mutant 


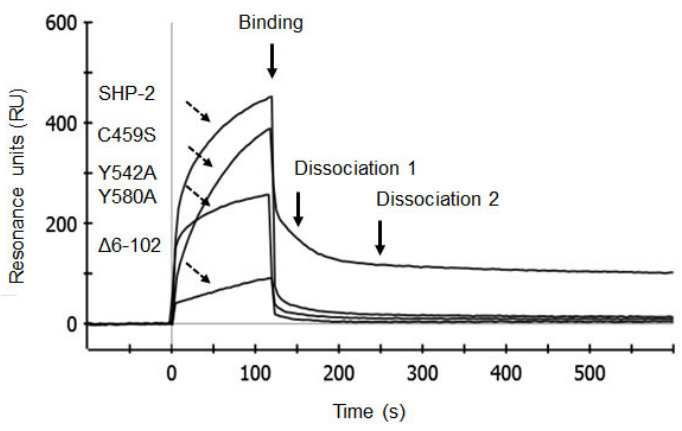

\begin{tabular}{|c|c|c|c|}
\hline & $\begin{array}{c}\text { Binding } \\
\text { (RU) }\end{array}$ & $\begin{array}{c}\text { Dissociation 1 } \\
\text { (RU) }\end{array}$ & $\begin{array}{c}\text { Dissociation 2 } \\
\text { (RU) }\end{array}$ \\
\hline SHP-2 & 446.04 & 33 & 18.41 \\
\hline$\Delta 6-102$ & 88.34 & 8.37 & 3.49 \\
\hline C459S & 383.57 & 167.13 & 117.49 \\
\hline Y542AY580A & 255.93 & 19.17 & 10.4 \\
\hline
\end{tabular}

Fig. 3. SPR analysis of wild-type and mutant SHP-2 binding to p-caveolin-1. Surface plasmon resonance (SPR) was used to examine binding kinetics of wild-type and mutant SHP-2 to p-caveolin-1 peptides. Caveolin-1 peptide was immobilized on the sensor chip and each recombinant SHP-2 protein was injected. Equilibrium binding curves and sensorgrams are shown. Three reference points used to measure the compound binding and dissociation (dissociation 1 and dissociation 2) are expressed in response units (RU). Experiments were repeated twice and both showed similar results.
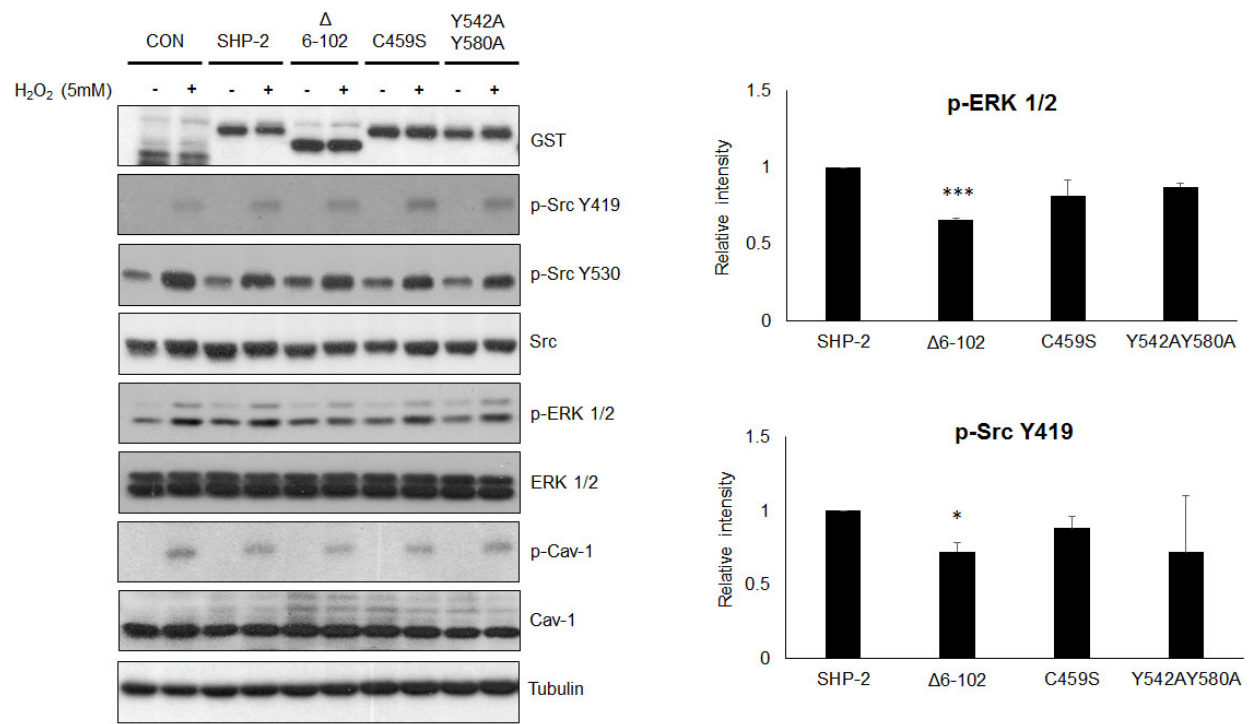

Fig. 4. Deletion of the $\mathrm{N}-\mathrm{SH} 2$ domain of SHP-2 (SHP-2 $\Delta 6-102)$ reduced $\mathrm{H}_{2} \mathrm{O}_{2}$-mediated Src phosphorylation at Tyr 419. Rat primary astrocytes that were transfected with GST, GST-SHP-2, GST-SHP-2 $\Delta 6-102$, GST-SHP-2 C459S, or GST-SHP-2 Y542AY580A for $4 \mathrm{~h}$ using the Pro-Ject Protein Transfection Reagent Kit were treated with $5 \mathrm{mM} \mathrm{H} \mathrm{H}_{2}$ for $10 \mathrm{~min}$, and then whole-cell lysates were extracted and subjected to immunoblotting. Blots were probed with anti-GST, anti-p-Src Y419, anti-p-Src Y530, anti-Src, anti-ERK 1/2, anti-p-ERK 1/2, anti-caveolin-1, anti-p-caveolin-1, and anti-tubulin antibodies. Tubulin was evaluated as a loading control. Data are representative of at least three experiments. Densitometric quantification of p-Src Y419 and p-ERK 1/2 levels, normalized to the level of GST. Values are means \pm SD from three experiments. ${ }^{*} \mathrm{P}<0.05,{ }^{* * *} \mathrm{P}<0.001$.

SHP-2 proteins for $4 \mathrm{~h}$, treated with or without $\mathrm{H}_{2} \mathrm{O}_{2}$ for 10 min, and then analyzed by immunoblotting with anti-GST, anti-p-Src419, anti-p-Src530, anti-Src, anti-ERK 1/2, anti-p-ERK 1/2, anti-caveolin-1, anti-p-caveolin-1, and anti-tubulin antibodies (Fig. 4). The transfection efficiency of wild-type and mutant SHP-2 protein was confirmed by the detected level of GST. Transfection of the N-SH2 domain deletion mutant of SHP-2 reduced the phosphorylation level of Src at Tyr 419 and
ERK $1 / 2$ under $\mathrm{H}_{2} \mathrm{O}_{2}$ treatment, compared with the wild-type SHP-2 transfection, in primary astrocytes. However, no significant change was detected for Tyr 530 phosphorylation of Src, with no change in the amount of Src or tubulin.

\section{DISCUSSION}

SHP-2, a member of protein-tyrosine phosphatases subfamily, 
is known to be highly expressed in specific brain regions, including the cortex, cerebellum, and hippocampus (21). Our previous studies demonstrated a positive role of SHP-2 in regulating Src activity through interaction with caveolin-1 in $\mathrm{H}_{2} \mathrm{O}_{2}$-mediated oxidative stress in brain astrocytes. In this study, to examine the region of SHP-2 responsible for the binding of caveolin-1, we examined the binding affinity of SHP-2 to caveolin-1 using wild-type and mutant SHP-2 proteins and the alteration of Src kinase activity following the transfection of wild-type and mutant SHP-2 under $\mathrm{H}_{2} \mathrm{O}_{2}$-mediated oxidative stress.

SHP-2 contains two SH2 domains, a phosphatase domain, and a C-terminal tail that contains two tyrosyl phosphorylation sites (12). The $\mathrm{SH} 2$ domains of SHP-2, especially $\mathrm{N}-\mathrm{SH} 2$, are involved in the engagement of bisphosphorylated ligands (12). Mutations in the SHP-2 PTP domain such as SHP-2 T466A and Y542AY580A, abolish the phosphatase activity $(22,23)$. The C-terminal tyrosine residues are phosphorylated by receptor and non-receptor protein-tyrosine kinases and this phosphorylation is required for the adaptor function of SHP-2, which contributes to the regulation of its phosphatase activity in multiple signaling pathways (24). Moreover, inhibition of SHP-2 results in reduced survival and increased neuronal cell death during nitric oxide exposure (6). These results support a neuroprotective role for SHP-2 against ischemic brain injury.

To determine which domain of SHP-2 is responsible for the binding of caveolin-1 and for modulating downstream signal transduction in response to ROS-induced oxidative stress, we systematically deleted or mutated SHP-2 and compared the binding of wild-type and mutant SHP-2s using flow cytometric competitive binding assays and SPR. Deletion of the $\mathrm{N}-\mathrm{SH} 2$ domain of SHP-2 (SHP-2 $\Delta 6$-102), showed reduced binding for caveolin-1, and reduced $\mathrm{H}_{2} \mathrm{O}_{2}$-mediated ERK phosphorylation and Src phosphorylation at Tyr 419 compared with the wild-type in rat primary astrocytes. The binding affinity of the Y542AY580A protein was also largely reduced compared with that of wild-type SHP-2. The point mutations in the tyrosine phosphatase domain of SHP-2 did not affect the binding kinetics to p-caveolin-1. These findings indicate that SHP-2 forms a complex with caveolin-1 and that complex formation between Tyr 14 residue of caveolin-1 and SHP-2 is dependent on the $\mathrm{N}-\mathrm{SH} 2$ domain of SHP-2 and phosphorylation status of the C-terminal tyrosine residues, but not the tyrosine phosphatase domain.

In this study, we provide initial evidence that the $\mathrm{N}-\mathrm{SH} 2$ domain and phosphorylation of the C-terminal tyrosine residues of SHP-2 play key roles in $\mathrm{H}_{2} \mathrm{O}_{2}$-induced caveolin-1/SHP-2 complex formation. These results suggest that SHP-2 functions mainly as an adaptor protein for the binding of caveolin-1 and contributes to the regulation of Src activity against ROS-induced oxidative stress in brain astrocytes. The role of a positive or negative regulator in cell signaling pathways of SHP-2 have been proposed by functioning as an adaptor protein (25). Negative regulatory effects of SHP-2 have been reported in that SHP-2 induces dephosphorylation of tyrosine-phosphorylated molecules, such as JAK/STATs (26). However, positive regulatory functions, by functioning as an adaptor protein, have also been demonstrated, as seen with interleukin-2 and epidermal growth factor, which result in the activation of downstream signaling pathways $(27,28)$. Our results suggest that the function of SHP-2 in brain astrocytes against $\mathrm{H}_{2} \mathrm{O}_{2}$ mediated oxidative stress seems to be a positive regulator in Src signaling pathways.

\section{MATERIALS AND METHODS}

Material and Methods are described in the online data supplement, available at http://www.bmbreports.org/.

\section{ACKNOWLEDGEMENTS}

This work was supported by a National Research Foundation of Korea (NRF) grant, funded by the Korean government (MSIP) (Grant 2012R1A5A2A32671866 to YHC) and a NRF-2014R1A1A1006593 (to HJP).

\section{REFERENCES}

1. Emerit J, Edeas M and Bricaire F (2004) Neurodegenerative diseases and oxidative stress. Biomed Pharmacother 58, 39-46

2. Reynolds A, Laurie C, Mosley RL and Gendelman HE (2007) Oxidative stress and the pathogenesis of neurodegenerative disorders. Int Rev Neurobiol 82, 297-325

3. Groeger G, Quiney C and Cotter TG (2009) Hydrogen peroxide as a cell-survival signaling molecule. Antioxid Redox Signal 11, 2655-2671

4. Uttara B, Singh AV, Zamboni P and Mahajan RT (2009) Oxidative stress and neurodegenerative diseases: a review of upstream and downstream antioxidant therapeutic options. Curr Neuropharmacol 7, 65-74

5. Hirrlinger J and Dringen R (2010) The cytosolic redox state of astrocytes: Maintenance, regulation and functional implications for metabolite trafficking. Brain Res Rev 63, 177-188

6. Park SJ, Lee JH, Kim HY et al (2012) Astrocytes, but not microglia, rapidly sense $\mathrm{H}(2) \mathrm{O}(2)$ via STAT6 phosphorylation, resulting in cyclooxygenase-2 expression and prostaglandin release. J Immunol 188, 5132-5141

7. Parpura V, Heneka MT, Montana V et al (2012) Glial cells in (patho)physiology. J Neurochem 121, 4-27

8. Tiffany-Castiglioni E, Hong S and Qian Y (2011) Copper handling by astrocytes: insights into neurodegenerative diseases. Int J Dev Neurosci 29, 811-818

9. Gee CE and Mansuy IM (2005) Protein phosphatases and their potential implications in neuroprotective processes. Cell Mol Life Sci 62, 1120-1130

10. Yun JH, Park SJ, Jo A et al (2011) Caveolin-1 is involved in reactive oxygen species-induced SHP-2 activation in astrocytes. Exp Mol Med 43, 660-668

11. Jo A, Park H, Lee SH et al (2014) SHP-2 binds to cav- 
eolin-1 and regulates Src activity via competitive inhibition of CSK in response to $\mathrm{H}_{2} \mathrm{O}_{2}$ in astrocytes. PLoS One 9, e91582

12. Neel BG, Gu H and Pao L (2003) The 'Shp'ing news: SH2 domain-containing tyrosine phosphatases in cell signaling. Trends Biochem Sci 28, 284-293

13. Kim HY, Park SJ, Joe EH and Jou I (2006) Raft-mediated Src homology 2 domain-containing proteintyrosine phosphatase 2 (SHP-2) regulation in microglia. J Biol Chem 281, 11872-11878

14. Lacalle RA, Mira E, Gomez-Mouton C, Jimenez-Baranda S, Martinez AC and Manes S (2002) Specific SHP-2 partitioning in raft domains triggers integrin-mediated signaling via Rho activation. J Cell Biol 157, 277-289

15. Pluskota E, Chen Y and D'Souza SE (2000) Src homology domain 2-containing tyrosine phosphatase 2 associates with intercellular adhesion molecule 1 to regulate cell survival. J Biol Chem 275, 30029-30036

16. Kim BW, Lee CS, Yi JS et al (2010) Lipid raft proteome reveals that oxidative phosphorylation system is associated with the plasma membrane. Expert Rev Proteomics 7, 849-866

17. Goetz JG, Lajoie P, Wiseman SM and Nabi IR (2008) Caveolin-1 in tumor progression: the good, the bad and the ugly. Cancer Metastasis Rev 27, 715-735

18. Cao H, Courchesne WE and Mastick CC (2002) A phosphotyrosine-dependent protein interaction screen reveals a role for phosphorylation of caveolin-1 on tyrosine 14: recruitment of C-terminal Src kinase. J Biol Chem 277, 8771-8774

19. Couet J, Li S, Okamoto T, Ikezu T and Lisanti MP (1997) Identification of peptide and protein ligands for the caveolin-scaffolding domain. Implications for the interaction of caveolin with caveolae-associated proteins. J Biol Chem 272, 6525-6533
20. Razani B, Woodman SE and Lisanti MP (2002) Caveolae: from cell biology to animal physiology. Pharmacol Rev 54, 431-467

21. Suzuki T, Matozaki T, Mizoguchi A and Kasuga M (1995) Localization and subcellular distribution of SH-PTP2, a protein-tyrosine phosphatase with Src homology-2 domains, in rat brain. Biochem Biophys Res Commun 211, 950-959

22. Merritt R, Hayman MJ and Agazie YM (2006) Mutation of Thr466 in SHP2 abolishes its phosphatase activity, but provides a new substrate-trapping mutant. Biochim Biophys Acta $1763,45-56$

23. Agazie YM and Hayman MJ (2003) Development of an efficient "substrate-trapping" mutant of Src homology phosphotyrosine phosphatase 2 and identification of the epidermal growth factor receptor, Gab1, and three other proteins as target substrates. J Biol Chem 278, 13952-13958

24. Poole AW and Jones ML (2005) A SHPing tale: perspectives on the regulation of SHP-1 and SHP-2 tyrosine phosphatases by the C-terminal tail. Cell Signal 17, 1323-1332

25. Salmond RJ and Alexander DR (2006) SHP2 forecast for the immune system: fog gradually clearing. Trends Immunol 27, 154-160

26. You M, Yu DH and Feng GS (1999) Shp-2 tyrosine phosphatase functions as a negative regulator of the interferon-stimulated Jak/STAT pathway. Mol Cell Biol 19, 2416-2424

27. Arnaud M, Mzali R, Gesbert F et al (2004) Interaction of the tyrosine phosphatase SHP-2 with Gab2 regulates Rho-dependent activation of the c-fos serum response element by interleukin-2. Biochem J 382, 545-556

28. Cai T, Nishida K, Hirano T and Khavari PA (2002) Gab1 and SHP-2 promote Ras/MAPK regulation of epidermal growth and differentiation. J Cell Biol 159, 103-112 